\title{
Numerical Analysis of a Miniature Microwave-discharge Ion Thruster Using Water as the Propellant*
}

\author{
Kengo NaKamura, ${ }^{1)}$ Yuichi NaKagawa, ${ }^{2)}$ Hiroyuki KoIzumi, ${ }^{3)}$ and Yoshinori TaKaO ${ }^{4) \dagger}$ \\ ${ }^{1)}$ Department of Systems Integration, Yokohama National University, Yokohama, Kanagawa 240-8501, Japan \\ ${ }^{2)}$ Department of Aeronautics and Astronautics, The University of Tokyo, Tokyo 113-8656, Japan \\ ${ }^{3)}$ Department of Advanced Energy, The University of Tokyo, Kashiwa, Chiba 277-8561, Japan \\ ${ }^{4)}$ Division of Systems Research, Yokohama National University, Yokohama, Kanagawa 240-8501, Japan
}

\begin{abstract}
We have conducted three-dimensional particle-in-cell simulations with Monte Carlo collisions method (PIC/MCC) to investigate the discharge characteristics of water for a microwave-discharge ion thruster, which can replace a high-pressure gas storage system. In the calculation model, three ion species $\left(\mathrm{H}_{2} \mathrm{O}^{+}, \mathrm{OH}^{+}\right.$, and $\left.\mathrm{H}^{+}\right)$are taken into account. The PIC/ MCC results indicate that the electron density, the potential, and the electron temperature are the highest near the ECR region, and in order to obtain a similar electron density, high absorbed power is required compared to the results using xenon as the propellant. For the ion composition ratio of the water discharges, $\mathrm{H}_{2} \mathrm{O}^{+}$and $\mathrm{OH}^{+}$dominate over $\mathrm{H}^{+}$and occupy more than $97 \%$. However, about $10 \%$ of the ion current density is derived from $\mathrm{H}^{+}$because of its lightweight and the effect of hydrogen ions would not be negligible for ion beam extraction.
\end{abstract}

Key Words: Propulsion, Ion Thrusters, PIC/MCC, Water, CubeSat

\author{
Nomenclature \\ $B$ : magnetic field \\ $E$ : electric field \\ $\phi$ : electrostatic potential \\ $\rho$ : charge density \\ $\varepsilon_{0}$ : permittivity of free space \\ $\varepsilon_{\mathrm{r}}$ : relative permittivity \\ $\mu_{0}$ : magnetic permeability of free space \\ $j$ : plasma current density \\ $m$ : mass \\ $P$ : power \\ $q$ : charge \\ $v$ : velocity \\ $x$ : position \\ $t$ : time \\ $n$ : density \\ $T_{\mathrm{e}}$ : electron temperature \\ $V$ : voltage \\ I: current \\ $\eta$ : efficiency \\ Subscripts \\ ES: electrostatic \\ EM: electromagnetic \\ ST: magnetostatic \\ abs: absorption \\ e: electron \\ i: ion
}

(C) 2018 The Japan Society for Aeronautical and Space Sciences *Presented at the 31st International Symposium on Space Technology and Science, 3-9 June 2017, Matsuyama, Japan.

Received 18 July 2017; final revision received 11 March 2018; accepted for publication 20 March 2018.

†Corresponding author, takao-yoshinori-yk@ynu.jp mul: multiply charged ion

div: divergence

S: screen

b: beam

\section{Introduction}

Commercial use of CubeSats has increased recently because the development costs and prelaunch preparation time can be reduced. Even deep space exploration missions can be carried out by CubeSats ${ }^{1)}$ or microspacecraft. ${ }^{2)}$ However, CubeSats have limits of weight, volume, and power, and thus, thrusters mounted on them must be miniaturized significantly. Ion thrusters are one of the candidates for a small and high-performance propulsion system. The research of a miniature ion propulsion system (MIPS) for 50-kg-class microspacecraft such as HODOYOSHI-4 was conducted in Japan and its operation in space was successfully performed in 2014 for the first time in the world. The MIPS employs electron cyclotron resonance (ECR) discharges with ring-shaped permanent magnets for its ion source and neutralizer. ${ }^{3-5}$ ) Since the MIPS utilizes xenon as the propellant, just as conventional ion thrusters, the high-pressure gas storage and feed system comprise most of the weight of the total propulsion system, which is difficult to miniaturize further. To remove the gas storage system, a small ion thruster using water as a liquid propellant has been proposed. Although water propellant has some problems, such as low-performance and oxidation characteristics, water also has the strong advantages of storage, safety, easy handling and availability compared to other propellants, which is important for microspacecraft. Moreover, oxidation is not a critical issue for the MIPS because it does not use a hollow cathode or electrodes for its ion source and neutralizer. The preliminary operation 
of the thruster has already been demonstrated at the University of Tokyo, ${ }^{6)}$ while the details of the discharge characteristics are not well known yet. Since it is difficult to perform plasma diagnostics and to identify what ion species are dominant in a small discharge chamber, we analyzed discharge characteristics of water using three-dimensional particle simulations in this study. Dependence on the power absorbed and influence of ionized ions are discussed. A comparison of the simulation results between water and xenon propellants, which was investigated in a previous study, ${ }^{7)}$ is also conducted.

\section{Numerical Model}

The numerical methodology is basically the same as that employed in our previous paper ${ }^{7)}$ except for the propellant, grid spacing, and time step; and thus, the model is briefly described below.

\subsection{Configuration}

Figure 1 shows the flow chart of the simulations. We employed a three-dimensional particle-in-cell method with Monte Carlo collisions (PIC/MCC) to analyze the kinetics of charged particles, together with the finite-difference time-domain method (FDTD) for the microwave electromagnetic field $\boldsymbol{E}_{\mathrm{EM}}$, and finite element analysis using ANSYS Emag $^{\mathrm{TM}}$ software for the magnetostatic field of the permanent magnets $\boldsymbol{B}_{\mathrm{ST}}$.

Figure 2 shows the calculation model, where the size of the discharge chamber is $20 \times 20 \times 4 \mathrm{~mm}^{3}$ with a ringshaped antenna located at $z=1.0 \mathrm{~mm}$. The inside of the coaxial waveguide is filled with the dielectric of boron nitride (BN). The $4.2 \mathrm{GHz}$ microwave power is fed to the ring antenna through the four spokes. It should be noted that the BN region is also included in the electromagnetic field calculation of microwaves, although the simulation area for the three-dimensional motion of charged particles is only the plasma region. Furthermore, the part of $\mathrm{BN}$ is in the center of the model as shown in Fig. 2, and the plasma density in this region is significantly small. ${ }^{7}$ Therefore, the effect of charging seems to have little effect on the discharge charac-

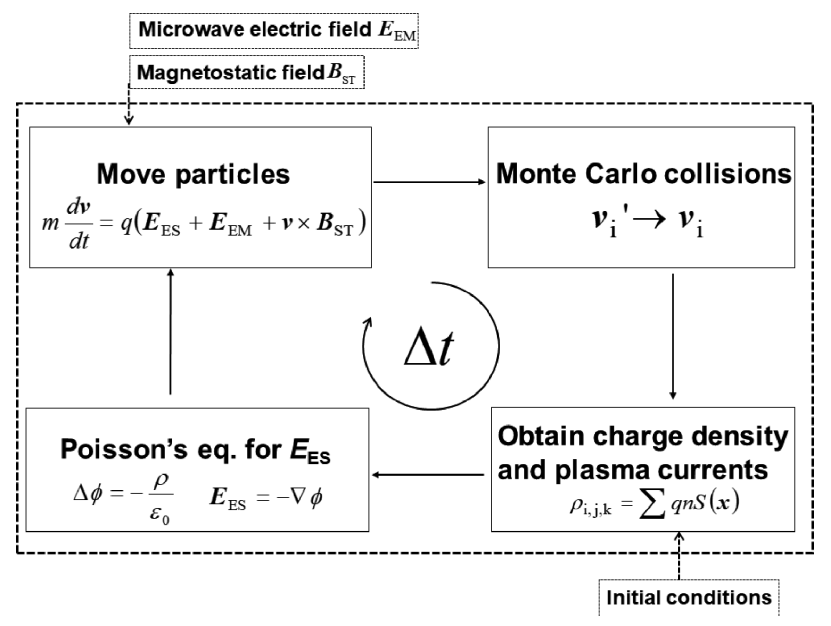

Fig. 1. Calculation flow of the PIC/MCC simulation. teristics. In order to reduce the calculation time, we conducted the simulation for only one-quarter of the region of the calculation model, as shown in Fig. 2. Figure 3 shows contour plots of the strength of the magnetic field of the ring-shaped permanent magnets in the $z-y$ plane. The thick lines in red represent the resonant magnetic field of $0.15 \mathrm{~T}$ for $4.2 \mathrm{GHz}$ microwaves.

\subsection{Numerical assumptions}

The present model has the following assumptions.

i) Singly-ionized water $\mathrm{H}_{2} \mathrm{O}^{+}$, hydroxide $\mathrm{OH}^{+}$, hydrogen $\mathrm{H}^{+}$, and electrons are treated as particles.

ii) Neutral particles are spatially and temporally uniform with a Maxwellian distribution at a gas temperature of $300 \mathrm{~K}$ in the plasma region.

iii) The magnetic fields of microwaves are negligibly small compared to the magnetostatic fields $\boldsymbol{B}_{\mathrm{ST}}$.

iv) The motion of excited-state atoms is not considered.

v) Coulomb collisions are not taken into account.

vi) Since the power of microwaves is low in this simula-

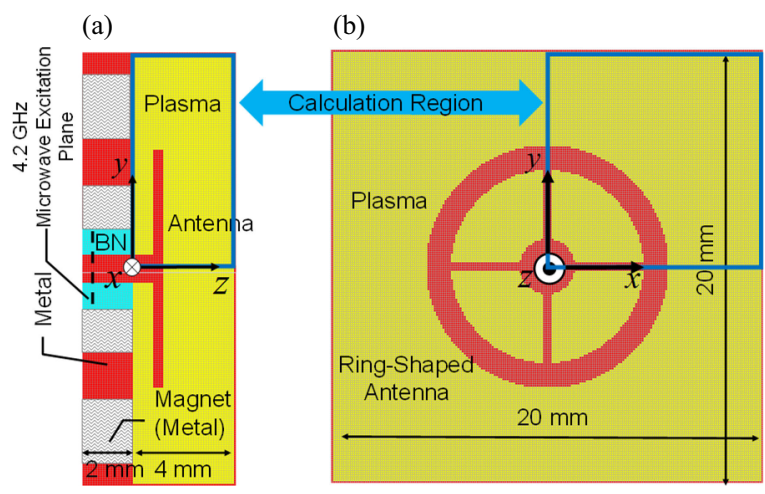

Fig. 2. Schematic diagrams of the calculation model: (a) Side view $(z-y / x$ plane at $x / y=0 \mathrm{~mm}$ ) and (b) front view $(x-y$ plane at $z=1.0 \mathrm{~mm})$. The red, yellow, and cyan regions represent the metal, plasma, and dielectric region, respectively. In order to reduce calculation time, we have conducted the simulation only for a quarter region using a point symmetry with respect to the $z$ axis.

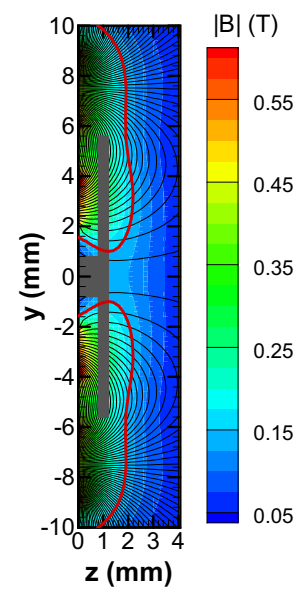

Fig. 3. Contour plots in the $z-y$ plane of the strength of the magnetic field of the ring-shaped permanent magnets, together with the thick lines in red representing the resonant magnetic field of $0.15 \mathrm{~T}$ for $4.2 \mathrm{GHz}$ microwaves.

(C IOP Publishing. Reproduced with permission. All rights reserved.) ${ }^{7)}$ 
Trans. Japan Soc. Aero. Space Sci., Vol. 61, No. 4, 2018

Table 1. Electron-neutral reactions of water considered in the present simulation.

\begin{tabular}{|c|c|c|c|c|}
\hline Label & Type of collision & Reaction & Threshold energy $(\mathrm{eV})$ & Ref. \\
\hline Elastic & Elastic scattering & $\mathrm{H}_{2} \mathrm{O}+\mathrm{e} \rightarrow \mathrm{H}_{2} \mathrm{O}+\mathrm{e}$ & & 9) \\
\hline rot1 & Rotational excitation & $\mathrm{H}_{2} \mathrm{O}(J=0)+\mathrm{e} \rightarrow \mathrm{H}_{2} \mathrm{O}(J=1)+\mathrm{e}$ & $4.60 \times 10^{-3}$ & 9), 10) \\
\hline $\operatorname{rot} 3$ & Rotational excitation & $\mathrm{H}_{2} \mathrm{O}(J=0)+\mathrm{e} \rightarrow \mathrm{H}_{2} \mathrm{O}(J=3)+\mathrm{e}$ & $1.18 \times 10^{-2}$ & $9), 10)$ \\
\hline vib1 & Vibrational excitation & $\mathrm{H}_{2} \mathrm{O}(000)+\mathrm{e} \rightarrow \mathrm{H}_{2} \mathrm{O}(010)+\mathrm{e}$ & 0.198 & 9) \\
\hline vib2 & Vibrational excitation & $\mathrm{H}_{2} \mathrm{O}(000)+\mathrm{e} \rightarrow \mathrm{H}_{2} \mathrm{O}[(100)+(001)]+\mathrm{e}$ & 0.453 & 9) \\
\hline $\mathrm{H}_{2} \mathrm{O}^{+}$ & Ionization & $\mathrm{H}_{2} \mathrm{O}+\mathrm{e} \rightarrow \mathrm{H}_{2} \mathrm{O}^{+}+2 \mathrm{e}$ & 13.5 & 9) \\
\hline $\mathrm{OH}^{+}$ & Dissociative ionization & $\mathrm{H}_{2} \mathrm{O}+\mathrm{e} \rightarrow \mathrm{OH}^{+}+\mathrm{H}+2 \mathrm{e}$ & 18.1 & 9) \\
\hline $\mathrm{H}^{+}$ & Dissociative ionization & $\mathrm{H}_{2} \mathrm{O}+\mathrm{e} \rightarrow \mathrm{H}^{+}+\mathrm{OH}+2 \mathrm{e}$ & 16.9 & 9) \\
\hline
\end{tabular}

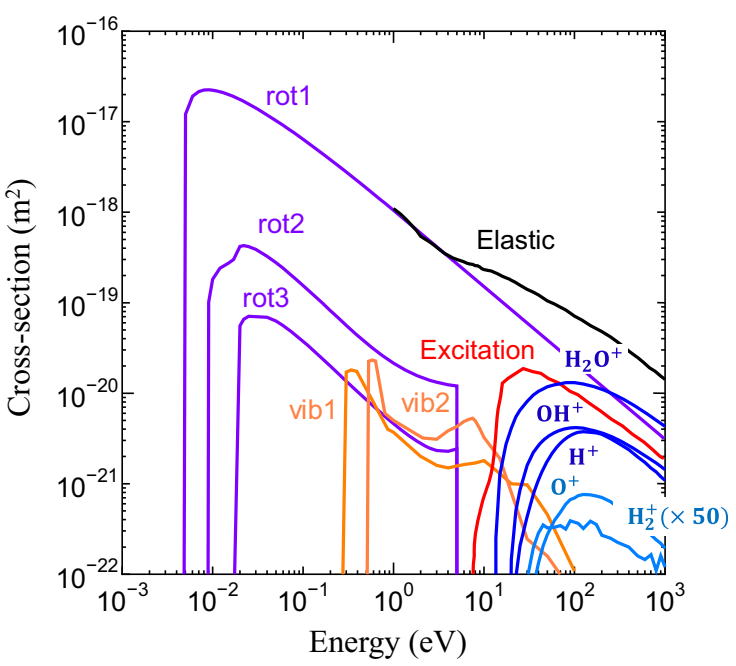

Fig. 4. Electron-neutral collision cross-sections of water.

tion, the plasma current is neglected. ${ }^{8)}$

\subsection{Collisions}

In electron-neutral collisions, the cross-sections for the dominant reactions used in this analysis are shown in Fig. 4, where these are compiled from Itikawa and Nigel, ${ }^{9)}$ Kawaguchi et al., ${ }^{10)}$ Yousfi and Benabdessadok, ${ }^{11)}$ and Petro and Sedwick. ${ }^{12)}$ In Fig. 4, excitation represents the sum of 26 types of the electronic excitations, which has a threshold energy of $7.14 \mathrm{eV}$. For a molecule like water, it is important to consider the energy dissipation mechanisms such as rotational and vibrational excitation collisions. In this analysis, rotational excitation at rotational transition $J=0 \rightarrow$ 1-3 and vibrational excitation at the stretching and bending mode, which is the recommended specific mode by Itikawa and Nigel, ${ }^{9)}$ are taken into account. In the ionized collisions, $\mathrm{H}_{2} \mathrm{O}^{+}, \mathrm{OH}^{+}$, and $\mathrm{H}^{+}$are considered as ionized particles, as you can see Fig. 4, since other ionized cross-sections are small compared with these ionized species such as $\mathrm{O}^{+}$and $\mathrm{H}_{2}{ }^{+}$. The electron-neutral reactions used in this analysis are listed in Table 1. Although negative ions should exist in water plasma, the cross-section of the attachment reactions generating negative ions is much smaller than that of ionization and other cross-sections. Accordingly, negative ions are secondary and are not taken into account in this analysis. In ion-neutral collisions, only charge exchange collisions are considered. ${ }^{13)}$

\subsection{Electrostatic field}

The electrostatic field $\boldsymbol{E}_{\mathrm{ES}}$ is given by

$$
\boldsymbol{E}_{\mathrm{ES}}=-\nabla \phi .
$$

The potential $\phi$ is derived from the space charge of charged particles. The Poisson's equation is given as

$$
\left(\frac{\partial^{2}}{\partial x^{2}}+\frac{\partial^{2}}{\partial y^{2}}+\frac{\partial^{2}}{\partial z^{2}}\right) \phi(x, y, z)=-\frac{\rho(x, y, z)}{\varepsilon_{0}} .
$$

Equation (2) is solved using the method of successive-overrelaxation (SOR) with boundary conditions of zero potential at all of the walls. Once the potential is obtained, the electrostatic field is determined using the central difference from the potential. To decrease the numerical noise, we apply digital smoothing to the space charge. ${ }^{14)}$

\subsection{Electromagnetic field}

The electromagnetic fields $\boldsymbol{E}_{\mathrm{EM}}$ of microwaves are obtained by solving Maxwell's equations using the FDTD method.

$$
\begin{gathered}
\nabla \times \boldsymbol{E}_{\mathrm{EM}}=-\frac{\partial \boldsymbol{B}}{\partial t}, \\
\nabla \times \boldsymbol{B}=\mu_{0}\left(\boldsymbol{j}+\varepsilon_{\mathrm{r}} \varepsilon_{0} \frac{\partial \boldsymbol{E}_{\mathrm{EM}}}{\partial t}\right) .
\end{gathered}
$$

We set the relative permittivity $\varepsilon_{\mathrm{r}}=1.0$ for the plasma, and $\varepsilon_{\mathrm{r}}=4.5$ for $\mathrm{BN}$.

\subsection{Motion and collisions of charged particles}

The equations of motion for charged particles are described as

$$
\begin{gathered}
\frac{d \boldsymbol{v}}{d t}=\frac{q}{m}\left(\boldsymbol{E}_{\mathrm{ES}}+\boldsymbol{E}_{\mathrm{EM}}+\boldsymbol{v} \times \boldsymbol{B}_{\mathrm{ST}}\right), \\
\frac{d \boldsymbol{x}}{d t}=\boldsymbol{v} .
\end{gathered}
$$

Equations (5) and (6) are solved using the leap-frog method and Buneman-Boris method. ${ }^{15)}$ In order to reduce calculation time, we employ the null-collision method in MCC. ${ }^{16)}$ The null-collision method utilizes a null-collision frequency that is constant with respect to energy. The collision probability becomes constant and there is no need to perform collision judgment for individual particles. A uniform random number is generated for all particles, and if that value is less than the collision frequency, the particle is regarded as collision fre- 

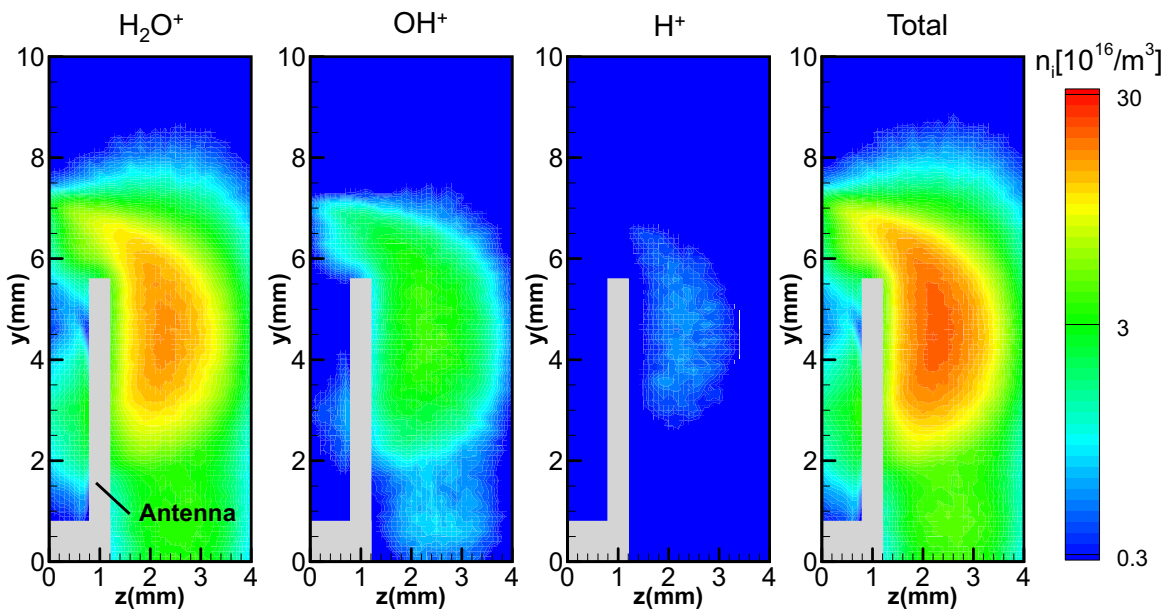

Fig. 5. Time-averaged distributions of each ion number density for $P_{\text {abs }}=2.0 \mathrm{~W}$ on the $z-y$ plane.

quency. After that, the uniform random number is generated for the collision particle, and the collision types such as elastic, excitation and ionization are determined. We use fully absorbing walls as the boundary condition for both electrons and ions, so that all electrons and ions disappear at the walls and antenna.

\subsection{Additional remarks}

In this simulation, the grid spacing is set at $0.1 \mathrm{~mm}$. The time step for FDTD is $\Delta t_{\mathrm{EM}}=1.49 \times 10^{-13} \mathrm{~s}(1 / 1600$ of a microwave cycle for $4.2 \mathrm{GHz}$ ). The time steps for PIC/ $\mathrm{MCC}$ are $\Delta t_{\mathrm{e}}=5.95 \times 10^{-12} \mathrm{~s}(1 / 40$ of a microwave cycle $)$ for electrons, and $\Delta t_{\mathrm{i}}=2.38 \times 10^{-10} \mathrm{~s}$ (one microwave cycle) for ions. In our previous study, ${ }^{7)}$ the pressure in the discharge chamber was set at 1 mTorr when xenon was injected as the propellant at the flow rate of $15 \mu \mathrm{g} / \mathrm{s}$, where the pressure value was obtained from Direct Simulation Monte Carlo (DSMC) simulation. Based on this assumption, corresponding to a mass flow rate of $35 \mu \mathrm{g} / \mathrm{s}$ in the experiment, ${ }^{6)}$ we set the neutral gas pressure in the discharge chamber at 6.3 mTorr using water as the propellant.

\section{Results and Discussion}

\subsection{Ion composition ratio}

Figure 5 shows the time-averaged distributions of ion number density for $\mathrm{H}_{2} \mathrm{O}^{+}, \mathrm{OH}^{+}, \mathrm{H}^{+}$, and the sum of them for $P_{\mathrm{abs}}=2.0 \mathrm{~W}$ at the $z-y$ plane on a logarithmic scale. Here, the results are averaged over 30,000 microwave cycles. The maximum value of $\mathrm{H}^{+}$number density is $0.6 \times 10^{16} / \mathrm{m}^{3}$ and much smaller than the other ion number densities. Since the composition ratio of each ion is $\mathrm{H}_{2} \mathrm{O}^{+}: \mathrm{OH}^{+}: \mathrm{H}^{+}=31: 7: 1$, $\mathrm{H}_{2} \mathrm{O}^{+}$is dominant for the ion species generated. This result is consistent with the ionized cross-sections as shown in Fig. 4. From the composition ratio of the ion number density, $\mathrm{H}_{2} \mathrm{O}^{+}$ and $\mathrm{OH}^{+}$densities occupy more than $97 \%$. From the experiment using the water ion thruster, ${ }^{17)}$ we found that the thrust coefficient is around 0.9 based on direct thrust measurement using a thrust stand, and is the same as compared to using xenon as the propellant. This result shows that most of the ex- tracted ion species are $\mathrm{H}_{2} \mathrm{O}^{+}$and $\mathrm{OH}^{+}$. This is because, if the $\mathrm{H}^{+}$is abundant in ion beam, the thrust coefficient drops more. The ion composition ratio estimated from the experiment is equivalent to that obtained in this study.

To estimate the ion beam profiles, there seems to be little adverse effect on thrust using water as the propellant because only minimal lightweight $\mathrm{H}^{+}$is generated and the difference in molecular weight between $\mathrm{H}_{2} \mathrm{O}^{+}$and $\mathrm{OH}^{+}$is just $1.0 \mathrm{~g} / \mathrm{mol}$, and therefore, there would be almost no difference in ion beam trajectories between $\mathrm{H}_{2} \mathrm{O}^{+}$and $\mathrm{OH}^{+}$. The ion current density related to the ion beam trajectories will be discussed later in Section 3.4.

One might imagine that hydroxyl radicals $(\mathrm{OH})$, which are highly reactive chemicals, are also generated and their generation results in chemical erosion of the thruster. However, for this experiment, the water ion thruster was operated for approximately $100 \mathrm{hr}$ in total, and there was no erosion detected on the grid electrode, microwave antenna or the discharge chamber, implying that it would be appropriate not to consider $\mathrm{OH}$ in the present study.

\subsection{Distributions of plasma parameters}

Figures 6(a)-6(c) and 6(d)-6(f) show the distributions of the electron density, the potential, and the electron temperature on the $z-y$ plane for $P_{\mathrm{abs}}=2.0$ and $3.0 \mathrm{~W}$, respectively. As the amount of power absorbed increases, the maximum values of the electron density and the potential increase. On the other hand, the maximal value of the electron temperature fluctuates only slightly. However, the area here the electron temperature is high somewhat expands downstream and towards the central axis. Peak values of the electron density are located at $z=2.2-2.3 \mathrm{~mm}$ near the ECR region, and the electron temperature is higher than $17 \mathrm{eV}$ in that area. Arcuate areas of the electron temperature more than $13 \mathrm{eV}$ are formed under both power conditions. This is because the electrons move along the magnetic field (see Fig. 3) lines as the result of being confined by the mirror magnetic fields, and the ionization reactions occur in the arcuate high-temperature region since the threshold energy of ionization is $13.5 \mathrm{eV}$. The plasma confinement results in distribution of 
(a) $2.0 \mathrm{~W}$

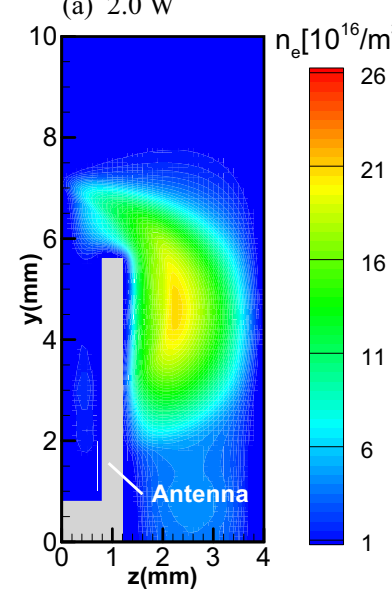

(d) $3.0 \mathrm{~W}$

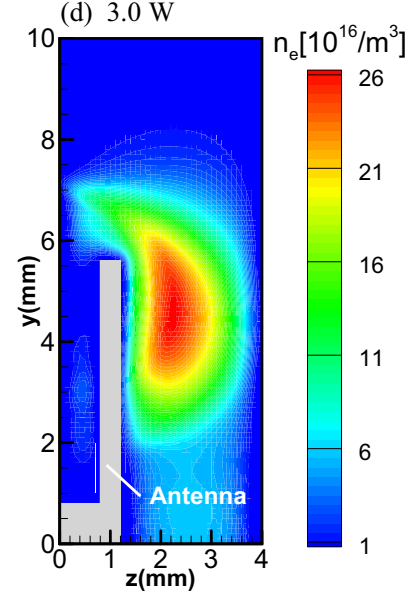

(b) $2.0 \mathrm{~W}$

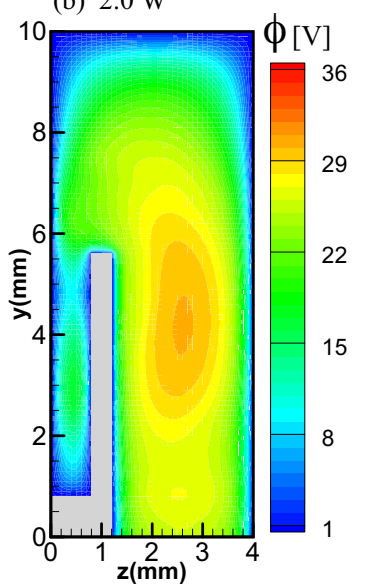

(e) $3.0 \mathrm{~W}$

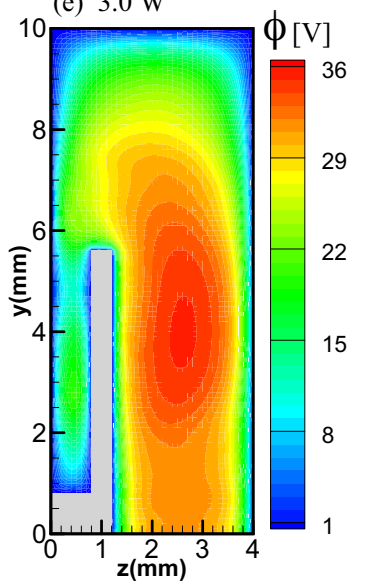

(c) $2.0 \mathrm{~W}$

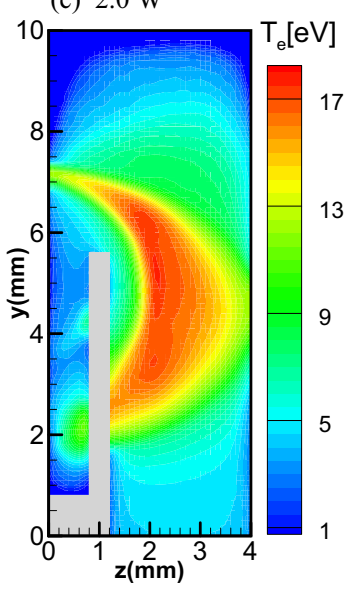

(f) $3.0 \mathrm{~W}$

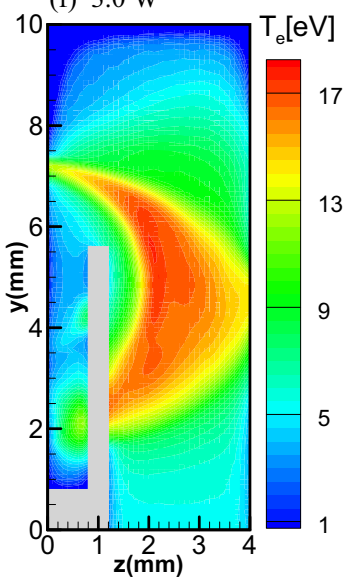

Fig. 6. Time-averaged distributions of (a) the electron density, (b) potential, and (c) electron temperature for $P_{\text {abs }}=2.0 \mathrm{~W}$; and (d) electron density, (e) potential, and (f) electron temperature for $P_{\mathrm{abs}}=3.0 \mathrm{~W}$ on the $z-y$ plane.

the plasma density as shown in Figs. 6(a) and 6(d). However, not all the electrons can be confined by the mirror magnetic field; some electrons of high energy are lost through a loss cone. Hence, increasing the amount of power absorbed expands the area of high electron temperature and does not increase the peak value, as can be seen from Figs. 6(c) and 6(f). It should be noted that the number of high-energy electrons in the ECR region tends to be overestimated and the resultant electron temperature also tends to be high because (electron-electron) Coulomb collisions are not taken into account, as described in Section 2.2 and in our previous paper. ${ }^{7)}$ If the effect of Coulomb collisions was considered, a somewhat lower electron temperature would be obtained owing to the relaxation of electron-electron collisions, as shown in Yamamoto et al. ${ }^{18)}$

We also investigated the time-varying distribution of the electron density. The results in this section were averaged over 100 microwave cycles. Figures 7(a) and 7(b) show the distributions of the electron density and time-averaged vectors of the electric field for $P_{\mathrm{abs}}=2.0 \mathrm{~W}$ in $z=2.2 \mathrm{~mm}$ of the $x-y$ plane, respectively, where the peak value of the electron density was observed on the $z-y$ plane. Figure 7(a) indicates that the electron density becomes the highest in the ECR region near the antenna having an outer diameter of $5.6 \mathrm{~mm}$. Furthermore, we found characteristic structures such as rotating striped patterns. As the amount of power absorbed increases, these characteristic structures appear more clearly. In addition, we found these structures in the distributions of the potential and the electron temperature. These stripped patterns and the time-averaged vectors of the electric field, such as those shown in Fig. 7(b), were also confirmed in a previous study, ${ }^{19)}$ and similar electron extraction can be expected in view of the plasma structure, where the azimuthal electric field and the radial magnetic field lead to electron transport across the magnetic field.

\subsection{Comparison with xenon results}

In a previous study, we used xenon as the propellant, ${ }^{7)}$ where we treated all regions with the grid spacing of $0.2 \mathrm{~mm}$. The time steps of FDTD and PIC/MCC were $\Delta t_{\mathrm{EM}}=2.98 \times 10^{-13} \mathrm{~s}(1 / 800$ of a microwave cycle for $4.2 \mathrm{GHz}), \Delta t_{\mathrm{e}}=5.95 \times 10^{-12} \mathrm{~s}$ for electrons and $\Delta t_{\mathrm{i}}=$ $2.38 \times 10^{-10} \mathrm{~s}$ for ions. Moreover, corresponding to a mass flow rate of $15 \mu \mathrm{g} / \mathrm{s}$ used under nominal condition for the xenon propellant, we set the neutral gas pressure in the discharge chamber at 1.0 mTorr. Figure 8 shows the distributions of the electron density, the potential, and the electron temperature of xenon on the $z-y$ plane for $P_{\mathrm{abs}}=0.7 \mathrm{~W} .^{7)}$

Comparing Figs. 6 and 8, more power absorption is re- 
(a) Distribution of electron density

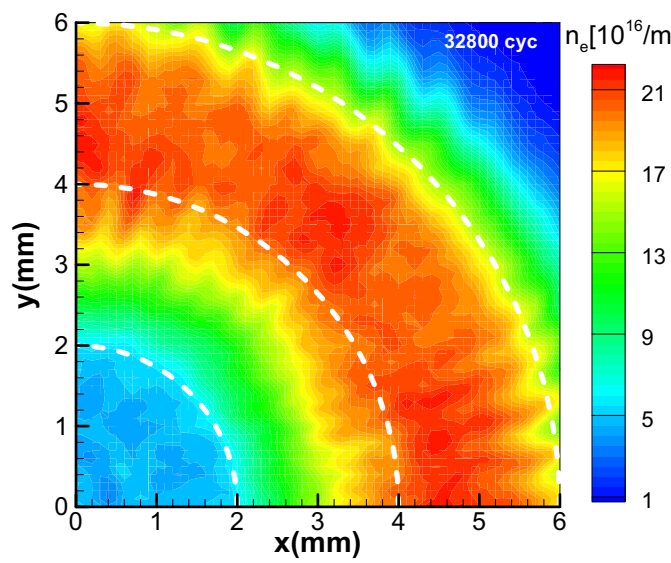

(b) Time-averaged vectors of electric field

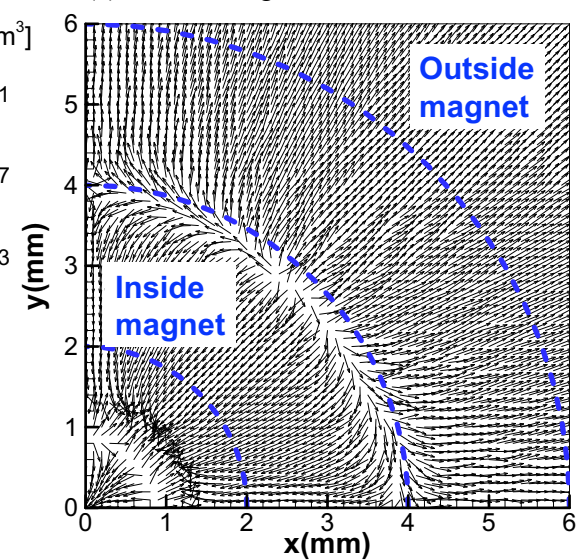

Fig. 7. (a) Distributions of the electron density and (b) the time-averaged vectors of electric field for $P_{\mathrm{abs}}=2.0 \mathrm{~W}$ at $z=2.2 \mathrm{~mm}$ of the $x-y$ plane. The dashed lines represent the boundaries of the permanent magnets.

(a) $0.7 \mathrm{~W}$

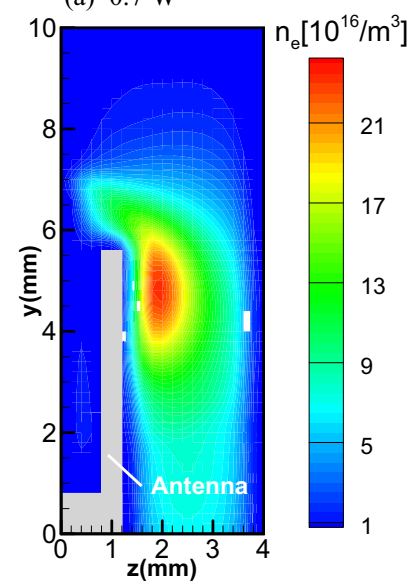

(b) $0.7 \mathrm{~W}$

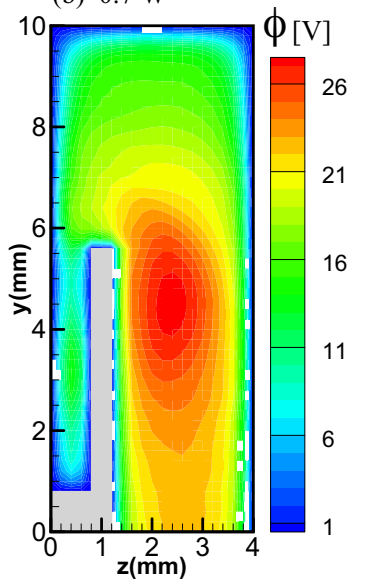

(c) $0.7 \mathrm{~W}$

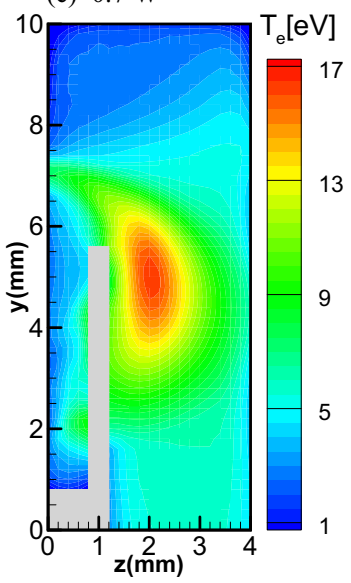

Fig. 8. Time-averaged distributions of (a) the electron density, (b) potential, and (c) electron temperature for $P_{\mathrm{abs}}=0.7 \mathrm{~W}$ on the $z-y$ plane of the xenon propellant.

quired when water is used as the propellant in order to obtain the same order of electron density as xenon. While xenon is a monoatomic molecule, water is a polyatomic molecule. As can be seen in Fig. 4, it is necessary to consider rotational and vibrational excitations, which cause a significant energy loss of electrons at low energy. Moreover, the ionization energy of water is $13.5 \mathrm{eV}$, which is larger than that of xenon, $12.1 \mathrm{eV}$. Hence, more power is required for water to maintain the plasma. Such a high power results in a strong electric field of microwaves, which increases the electron temperature. However, too high-energy electrons escape from the mirror magnetic confinement, and thus, the area of high electron temperature expands instead of increasing the peak electron temperature, as shown in Figs. 6(c) and 6(f).

\subsection{Ion current density}

We also considered the effects of ion species on thrust. The thrust of an ion thruster is estimated using Eq. (7)

$$
F=\eta_{\mathrm{mul}} \eta_{\mathrm{div}} \sqrt{\frac{2 m_{\mathrm{i}} V_{\mathrm{S}}}{q}} I_{\mathrm{b}} .
$$

For the water propellant, Eq. (7) indicates that $m_{\mathrm{i}}$ affects the thrust since various ions are generated, so the effect of each ion species was investigated. Figure 9 shows the distributions of the ion current density for $\mathrm{H}_{2} \mathrm{O}^{+}, \mathrm{OH}^{+}$, and $\mathrm{H}^{+}$in the $z$ direction for $P_{\mathrm{abs}}=2.0 \mathrm{~W}$ on the $z-y$ plane $(x=0 \mathrm{~mm})$. As can be seen, the direction of $j_{z}$ changed near $z=2.6 \mathrm{~mm}$, and almost all of the ions are lost on the wall and the antenna at $z=4.0 \mathrm{~mm}$. It should be noted that $j_{z}$ at $z=4.0 \mathrm{~mm}$ spreads over the entire area of the $x-y$ plane, although $j_{z}$ in the negative $z$ direction is concentrated on the ring-shaped antenna and its four spokes, as shown in Fig. 2. This configuration results in a larger absolute value for negative $j_{z}$.

Figure 10 shows the distribution of $\mathrm{H}_{2} \mathrm{O}^{+}$current density at $z=4.0 \mathrm{~mm}$ on the $x-y$ plane. The ion current density becomes the highest at the region where the highest electron number density is obtained. Although we did not consider the extraction of ions in this analysis, the average value of ion current density at $z=4.0 \mathrm{~mm}$, which is an estimation of the thrust, is shown in Table 2. These values are averaged between 0 and $7 \mathrm{~mm}$ in the $x$ and $y$ directions since the ion current flowing into those areas mainly contributes to the 

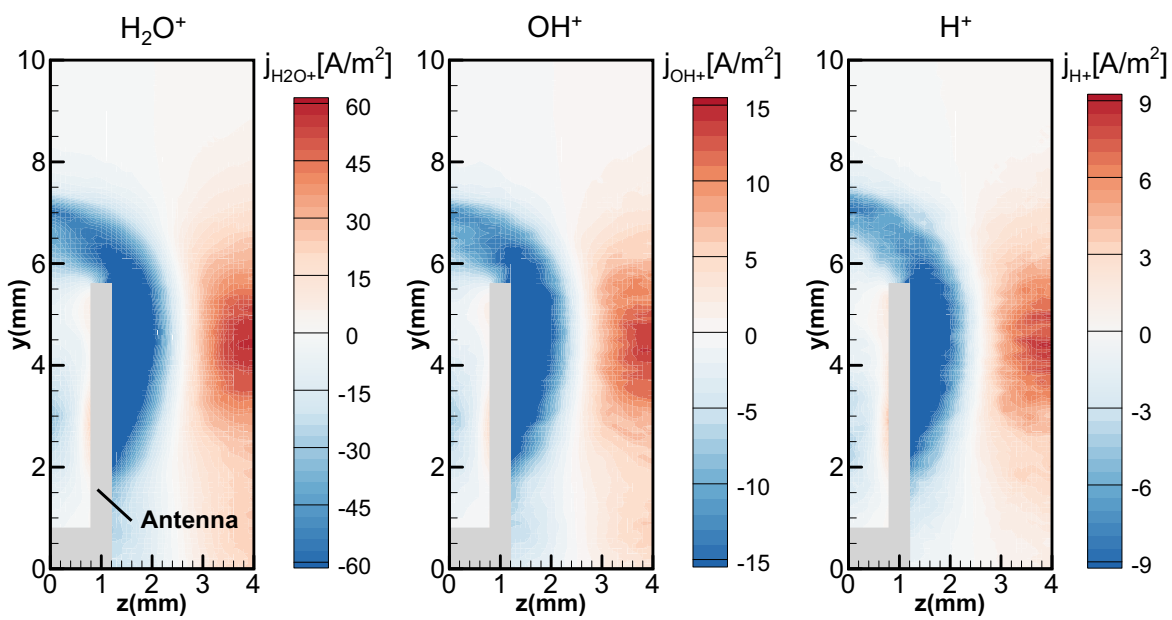

Fig. 9. Time-averaged distributions of the ion current density in the $z$ direction for $\mathrm{H}_{2} \mathrm{O}^{+}, \mathrm{OH}^{+}$, and $\mathrm{H}^{+}$for $P_{\text {abs }}=2.0 \mathrm{~W}$ on the $z-y$ plane.

Table 2. The averaged value of ion current density for each ion species at $z=4.0 \mathrm{~mm}$.

\begin{tabular}{cc}
\hline Ion species & $J_{z}$ averaged at $z=4.0 \mathrm{~mm}$ \\
\hline $\mathrm{H}_{2} \mathrm{O}^{+}$ & $30.8 \mathrm{~A} / \mathrm{m}^{2}$ \\
$\mathrm{OH}^{+}$ & $7.02 \mathrm{~A} / \mathrm{m}^{2}$ \\
$\mathrm{H}^{+}$ & $4.08 \mathrm{~A} / \mathrm{m}^{2}$ \\
\hline
\end{tabular}

thrust. Table 2 indicates that the ion current density of $\mathrm{H}_{2} \mathrm{O}^{+}$ is the largest among the ion species examined. Moreover, the ion current density of $\mathrm{H}^{+}$occupies approximately $10 \%$ of the total ion current density. Comparing the ion number density and ion current density, the ratio of $\mathrm{H}^{+}$current density increases owing to the high speed of lightweight $\mathrm{H}^{+}$. Furthermore, its contribution cannot be neglected, whereas the number density of $\mathrm{H}^{+}$is smaller than $3 \%$. Although $\mathrm{H}^{+}$has the advantage of specific impulse, the thrust is reduced in terms of thrust-to-power ratio in microspacecraft with limited power. Moreover, $\mathrm{H}^{+}$is not desirable because its molecular weight is light and the ion beam trajectory may change and collide with grid electrodes, and additional power absorption is required owing to the dissociation process of $\mathrm{H}_{2} \mathrm{O}$. The latter would result in low thruster efficiency. Therefore, it is believed the discharge condition to reduce the $\mathrm{H}^{+}$current density should be investigated or that a grid system fit for heavy $\mathrm{H}_{2} \mathrm{O}^{+}, \mathrm{OH}^{+}$and light $\mathrm{H}^{+}$is required.

\section{Conclusions}

In this study, we analyzed the discharge characteristics of water, which is employed as the propellant of a miniature microwave discharge ion thruster, using three-dimensional particle simulations ( $\mathrm{PIC} / \mathrm{MCC}$ ), where three ion species $\left(\mathrm{H}_{2} \mathrm{O}^{+}, \mathrm{OH}^{+}\right.$, and $\left.\mathrm{H}^{+}\right)$are considered. The PIC/MCC results show that the electron density, the potential, and the electron temperature have maximal values in the ECR region near the antenna. Compared to using xenon as the propellant, additional power absorption is required for water discharge in order to obtain the same electron density as xenon. In addition, we observed rotating striped patterns as seen in the xenon

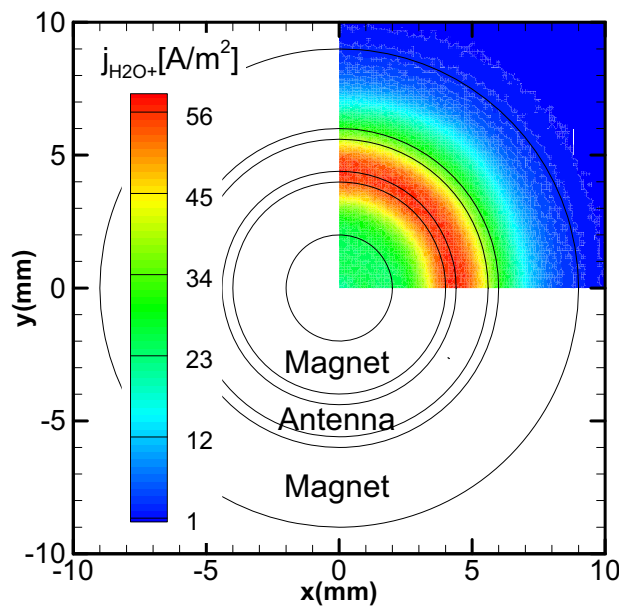

Fig. 10. Time-averaged distribution of the $\mathrm{H}_{2} \mathrm{O}^{+}$current density at $z=4.0 \mathrm{~mm}$ of the $x-y$ plane for $P_{\mathrm{abs}}=2.0 \mathrm{~W}$.

plasma discharge in a previous study, implying that electrons can be extracted if the plasma source is used as a neutralizer. Although the number density of $\mathrm{H}^{+}$is smaller than $3 \%$, the current density of $\mathrm{H}^{+}$exceeds $10 \%$, and thus, the effect of $\mathrm{H}^{+}$would not be negligible for ion beam extraction.

In future work, we will investigate the discharge conditions to reduce the ion current density of $\mathrm{H}^{+}$as the ion beam source. Negative ion species, such as $\mathrm{OH}^{-}, \mathrm{H}^{-}$and $\mathrm{O}^{-}$, will also be considered for an improved model in order to analyze water plasma in more detail.

\section{Acknowledgments}

This work was supported in part by JSPS KAKENHI Grant Number JP16H06370. Part of the computer simulation was performed on the KDK computer system at the Research Institute for Sustainable Humanosphere, Kyoto University.

\section{References}

1) Mars Cube One (MarCO) Mission Overview, NASA JPL, http: //www.jpl.nasa.gov/cubesat/missions/marco.php (accessed June 21, 2017). 
2) Koizumi, H., Kawahara, H., Yaginuma, K., Asakawa, J., Nakagawa, Y., Nakamura, Y., Kojima, S., Matsuguma, T., Funase, R., Nakatsuka, J., and Komurasaki, K.: Initial Flight Operations of the Miniature Propulsion System Installed on Small Space Probe: PROCYON, Trans. JSASS Aerospace Technology Japan, 14 (2016), pp. Pb_13-Pb_22.

3) Kuninaka, H., Nishiyama, K., Funaki, I., Yamada, T., Shimizu, Y., and Kawaguchi, J.: Powered Flight of Electron Cyclotron Resonance Ion Engines on Hayabusa Explorer, J. Propul. Power, 23 (2007), pp. 544-551.

4) Kuninaka, H. and Satori, S.: Development and Demonstration of a Cathodeless Electron Cyclotron Resonance Ion Thruster, J. Propul. Power, 14 (1998), pp. 1022-1026.

5) Koizumi, H. and Kuninaka, H.: Miniature Microwave Discharge Ion Thruster Driven by 1 Watt Microwave Power, J. Propul. Power, 26 (2010), pp. 601-604.

6) Nakagawa, Y., Kawahara, H., Koizumi, H., and Komurasaki, K.: Fundamental Experiments with Liquid Propellants for the Microwave-discharge Ion Thruster, Space Propulsion 2016, Roma, Italy, SP2016312540, 2016

7) Takao, Y., Koizumi, H., Komurasaki, K., Eriguchi, K., and Ono, K.: Three-dimensional Particle-in-cell Simulation of a Miniature Plasma Source for a Microwave Discharge Ion Thruster, Plasma Sources Sci. Technol., 23 (2014), pp. 064004-1-11.

8) Takao, Y., Eriguchi, K., Ono, K., Sugita, Y., Koizumi, H., and Komurasaki, K.: A Validation Study of a 3D PIC Model for a Miniature Microwave Discharge Ion Thruster, 50th AIAA/ASME/SAE/ ASEE Joint Propulsion Conf., AIAA-2014-3829, 2014.

9) Itikawa, Y. and Nigel, M.: Cross Sections for Electron Collisions with Water Molecules, J. Phys. Chem. Ref. Data, 34 (2005), pp. 1-22.

10) Kawaguchi, S., Takahashi, K., Satori, K., and Itoh, H.: Electron Transport Analysis in Water Vapor, Jpn. J. Appl. Phys., 55 (2016), pp. 07LD03-1-7.

11) Yousfi, M. and Benabdessadok, M. D.: Boltzman Equation Analysis of
Electron-molecule Collision Cross Sections in Water Vapor and Ammonia, J. Appl. Phys., 80 (1996), pp. 6619-6630.

12) Petro, E. M. and Sedwick, R. J.: Effects of Water-Vapor Propellant on Electrodeless Thruster Performance, J. Propul. Power, 33 (2017), pp. 1410-1417.

13) Lishawa, C. R., Dressler, R. A., Gardner, J. A., Salter, R. H., and Murad, E.: Cross Sections and Product Kinetic Energy Analysis of $\mathrm{H}_{2} \mathrm{O}^{+}-\mathrm{H}_{2} \mathrm{O}$ Collisions at Suprathermal Energies, J. Chem. Phys., 93 (1990), pp. 3196-3206.

14) Veboncoeur, J. P.: Particle Simulation of Plasmas: Review and Advances, Plasma Phys. Control Fusion, 47 (2005), pp. A231-A260.

15) Birdsall, C. K. and Langdon, A. B.: Plasma Physics via Computer Simulation, IOP Publishing, Bristol, 1991.

16) Vahedi, V. and Surendra, M.: A Monte Carlo Collision Model for the Particle-in-cell Method: Applications to Argon and Oxygen Discharges, Comput. Phys. Commun., 87 (1995), pp. 179-198.

17) Nakagawa, Y., Tomita, D., Koizumi, H., and Komurasaki, K.: Design and Test of a $100 \mu \mathrm{N}$-class Thrust Stand for a Miniature Water Ion Thruster with CubeSat, Trans. JSASS Aerospace Technology Japan (to be published).

18) Yamamoto, N., Tomita, K., Yamazaki, N., Tsuru, T., Ezaki, T., Kotani, Y., Uchino, K., and Nakashima, H.: Measurements of Electron Density and Temperature in a Miniature Microwave Discharge Ion Thruster using Laser Thomson Scattering Technique, Plasma Sources Sci. Technol., 19 (2010), pp. 045009-1-7.

19) Hiramoto, K., Nakagawa, Y., Koizumi, H., and Takao, Y.: Effects of $\mathrm{E} \times \mathrm{B}$ Drift on Electron Transport Across the Magnetic Field in a Miniature Microwave Discharge Neutralizer, Phys. Plasmas, 24 (2017), pp. 064504-1-5.

Kimiya Komurasaki Associate Editor 